\title{
A Spatial-Stiffness Analysis of Fiducial Registration Accuracy
}

\author{
Burton Ma and Randy E. Ellis \\ School of Computing, Queen's University at Kingston, Canada K7L 3N6
}

\begin{abstract}
We describe a new approach to predicting the maximum target registration error for fiducial registration. The approach is based on the analysis of a spatial stiffness model that produces an analytic expression of the maximum error that is closely related to the formula reported in Fitzpatrick et al.[1]. The methodology we used was developed by Lin et al. [4]. The analytic stiffness results yield error estimates that closely match those from numerical simulations.
\end{abstract}

\section{Introduction}

Fiducial markers, used to accurately localize an object in space, have many applications in the health sciences such as registration for computer-integrated surgery and radiotherapy, roentgen stereo photogrammetric analysis, and motion tracking in biomechanical studies. Understanding how measurement errors of the fiducials affect the accuracy of the localization is important for the clinical application of fiduciary techniques. An analytic expression of the expected target registration error (TRE) was derived by Fitzpatrick et al. [1] in which many important relationships were shown. Their derivation of the analytic expression is not trivial and does not easily lend itself to the case of surface-based registration.

We have approached the problem of estimating registration accuracy using the theory of mechanism stiffness. The fiducial markers are viewed as the points where an elastic suspension system is attached to a rigid mechanism. By analyzing the stiffness matrix of the mechanism using the techniques developed by Lin et al. [4], we are able to derive an analytic expression of the maximum TRE that is very similar to the expression derived by Fitzpatrick et al. [1]. We compare the predictions of this stiffness analysis to numerical simulations described by Maurer et al. [6].

\section{Background: Stiffness of a Passive Mechanical System}

A general model of the elastic behaviour of a passive (unactuated at the instant of analysis) mechanism is a rigid body suspended by linear and torsional springs. This model leads to the concept of spatial stiffness or compliance of the mechanism. The background material we give here, from the robotics literature, is based on Lin et al. [4] and is closely related to the compliant axes described by Patterson and Lipkin [10].

For a passive mechanism in local equilibrium, a twist displacement $\mathbf{t}$ of a rigid body is related to a counteracting wrench force $\mathbf{w}$ by a $6 \times 6$ spatial stiffness matrix $\mathbf{K}$ :

$$
\mathbf{w}=\mathbf{K} \mathbf{t}=\left[\begin{array}{ll}
\mathbf{A} & \mathbf{B} \\
\mathbf{B}^{T} & \mathbf{D}
\end{array}\right] \mathbf{t}
$$




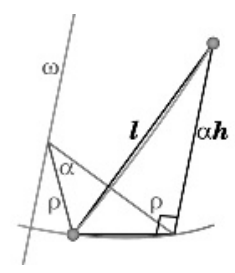

Fig. 1. The displacement of a point under a screw motion. The point rotates by an amount $\alpha$ about the axis with direction $\boldsymbol{\omega}$, then translates parallel to the axis by an amount $\alpha h$. The net distance of displacement is $l$.

where $\mathbf{A}, \mathbf{B}$, and $\mathbf{D}$ are $3 \times 3$ matrices. The twist is a vector $\mathbf{t}=\left[\boldsymbol{v}^{T} \boldsymbol{\omega}^{T}\right]^{T}$ where $\boldsymbol{v}^{T}=$ $\left[\begin{array}{lll}v_{x} & v_{y} & v_{z}\end{array}\right]$ is linear displacement and $\boldsymbol{\omega}^{T}=\left[\omega_{x} \omega_{y} \omega_{z}\right]$ is rotational displacement. The wrench is a vector $\mathbf{w}=\left[\mathbf{f}^{T} \boldsymbol{\tau}^{T}\right]^{T}$ where $\mathbf{f}^{T}=\left[f_{x} f_{y} f_{z}\right]$ is force and $\boldsymbol{\tau}^{T}=\left[\tau_{x} \tau_{y} \tau_{z}\right]$ is torque. If $\mathbf{K}$ is invertible then an applied wrench produces a twist displacement $\mathbf{t}=\mathbf{C w}$ where $\mathbf{C}=\mathbf{K}^{-1}$ represents the compliance matrix. Equation 1 is simply a general expression of Hooke's law. We can obtain $\mathbf{K}$ by evaluating the Hessian of the potential energy $U$ of the system at equilibriun 1 (Mishra and Silver [8]).

$\mathbf{K}$ is symmetric positive definite for stable springs and small displacements from equilibrium. The eigenvalues of $\mathbf{K}$ are not immediately useful because their magnitudes change depending on the coordinate frame used to define $\mathbf{K}$; however, there exists a subspace $V$ of twists given by

$$
V=\{\mathbf{t}=\mathbf{P} \boldsymbol{\omega}\} \quad \text { where } \quad \mathbf{P}=\left[\begin{array}{c}
-\mathbf{A}_{\mathbf{I}}^{-1} \mathbf{B} \\
\mathbf{I}
\end{array}\right]
$$

in which the twist, parameterized by rotational displacement, induces a pure torque. The torque can be computed as $\mathbf{K}_{V} \boldsymbol{\omega}$ where

$$
\mathbf{K}_{V}=\mathbf{D}-\mathbf{B}^{T} \mathbf{A}^{-1} \mathbf{B} .
$$

The eigenvalues $\mu_{1}, \mu_{2}, \mu_{3}$ of $\mathbf{K}_{V}$ are frame-invariant and are called the principal rotational stiffnesses. There is also a subspace $\mathcal{W}$ of wrenches parameterized by force where the wrench induces a pure translation. The translation is given by $\mathbf{C}_{\mathcal{W}} \mathbf{f}$ where $\mathbf{C}_{\mathcal{W}}=\mathbf{A}^{-1}$. The eigenvalues $\sigma_{1}, \sigma_{2}, \sigma_{3}$ of $\mathbf{C}_{\mathcal{W}}^{-1}$ are frame-invariant and are called the principal translational stiffnesses.

To compute TRE we need to be able to compute the distance that a point moves under a twist displacement. We do this by using the screw representation of the twist. A screw is rotation about an axis followed by a translation parallel to the axis. It is described by its pitch, $h$, which is the ratio of translational motion to rotational motion, and its magnitude, $M$, which is the net rotation. For a twist (Murray et al. [9]) $h=\boldsymbol{\omega} \cdot \boldsymbol{v} /\|\boldsymbol{\omega}\|^{2}, M=\|\boldsymbol{\omega}\|$, and the axis of the screw is parallel to $\boldsymbol{\omega}$ passing through the point $\mathbf{q}=\boldsymbol{\omega} \times \boldsymbol{v} /\|\boldsymbol{\omega}\|^{2}$; by convention, a pure translation (where $\boldsymbol{\omega}=\mathbf{0}$ ) has $h=\infty$ and $M=\|\boldsymbol{v}\|$, with the screw axis parallel to $\boldsymbol{v}$ passing through the origin. A unit twist has magnitude $M=1$, in which case, for $\boldsymbol{\omega} \neq \mathbf{0}, h=\boldsymbol{\omega} \cdot \boldsymbol{v}$ and $\mathbf{q}=\boldsymbol{\omega} \times \boldsymbol{v}$. For a small screw motion with

\footnotetext{
${ }^{1}$ This is especially easy to see in the case of a simple linear spring: $U(x)=k\left(x-x_{0}\right)^{2} / 2$, $d U / d x=k\left(x-x_{0}\right), d^{2} U / d x^{2}=k$.
} 

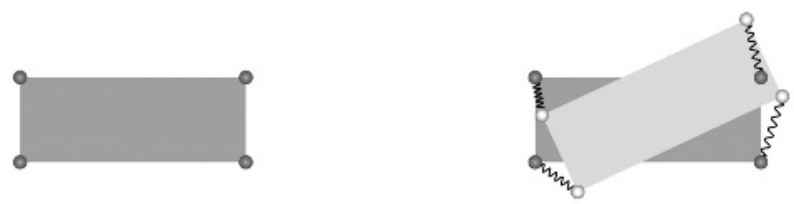

Fig. 2. Spatial stiffness model for $N=4$ fiducials. The fiducials in their original positions (black) are displaced to new positions (white) by a small rotation and translation. The sum of the squared distances is proportional to the energy stored in the linear springs connecting the displaced fiducials to their original locations.

$M=\alpha$ and $\boldsymbol{\omega} \neq 0$, a point located at a distance $\rho$ from the screw axis will be displaced by length (see Figure 1)

$$
\begin{array}{rrr}
l & =\sqrt{\rho^{2}+\rho^{2}-2 \rho^{2} \cos \alpha+(\alpha h)^{2}} & \text { (law of cosines) } \\
& \approx \sqrt{2 \rho^{2}-2 \rho^{2}\left(1-\alpha^{2} / 2\right)+\alpha^{2}(\boldsymbol{\omega} \cdot \boldsymbol{v})^{2}} & \text { (Maclaurin series approximation) } \\
& =|\alpha| \sqrt{\rho^{2}+(\boldsymbol{\omega} \cdot \boldsymbol{v})^{2}} &
\end{array}
$$

Equation 4 is also the basis of the frame-invariant quality measure for compliant grasps described by Lin et al. [4]. Because the principal rotational and translational stiffnesses have different units, they cannot be directly compared to one another. One solution is to scale the principal rotational stiffnesses by an appropriate factor (see Lin et al. [4] for details) to yield the so-called equivalent stiffnesses, $\mu_{\mathrm{eq}, i}$ :

$$
\mu_{\mathrm{eq}, i}=\mu_{i} /\left(\rho_{i}^{2}+\left(\boldsymbol{\omega}_{i} \cdot \boldsymbol{v}_{i}\right)^{2}\right) \quad i=1,2,3
$$

where, $\mu_{i}$ is an eigenvalue of $\mathbf{K}_{V}$ with an associated eigenvector $\boldsymbol{\omega}_{i}, \boldsymbol{v}_{i}=\mathbf{P} \boldsymbol{\omega}_{i}$, and $\rho_{i}$ is the distance between the point of interest and the screw axis of the twist $\left[\boldsymbol{v}_{i}^{T} \boldsymbol{\omega}_{i}^{T}\right]^{T}$. The equivalent stiffnesses can be compared to the principal translational stiffnesses which leads to the stiffness quality measure $Q=\min \left(\mu_{\mathrm{eq}, 1}, \mu_{\mathrm{eq}, 2}, \mu_{\mathrm{eq}, 3}, \sigma_{1}, \sigma_{2}, \sigma_{3}\right) . Q$ characterizes the least constrained displacement of the mechanism. Therefore, maximizing the smallest rotational and translational stiffnesses will minimize the worst-case displacement of the mechanism.

\section{Spatial Stiffness and Fiducial Registration}

The spatial stiffness model for fiducial registration is shown in Figure 2 The model parameters are $N$ fiducial markers with locations $\left\{\mathbf{p}_{i}\right\}$ for $i=1, \ldots N$. To simplify the analysis, we assume that the centroid of the fiducials coincides with the origin. Suppose each marker is displaced by a small rotation $\mathbf{R}=\mathbf{R}_{\mathbf{z}}\left(\omega_{z}\right) \mathbf{R}_{\mathbf{y}}\left(\omega_{y}\right) \mathbf{R}_{\mathbf{x}}\left(\omega_{x}\right)$ and small translation $\boldsymbol{\delta}=\left[\begin{array}{lll}t_{x} & t_{y} & t_{z}\end{array}\right]^{T}$. The locations $\mathbf{q}_{i}$ of the displaced markers are given by $\mathbf{q}_{i}=\mathbf{R} \mathbf{p}_{i}+\boldsymbol{\delta}$. Assuming a spring constant of one, the potential energy $U_{i}$ stored in each linear spring is $U_{i}=\frac{1}{2}\left(\mathbf{q}_{i}-\mathbf{p}_{i}\right)^{T}\left(\mathbf{q}_{i}-\mathbf{p}_{i}\right)$. Using symbolic computation software, it can be shown that the Hessian $\mathbf{H}_{i}$ of $U_{i}$ evaluated at equilibrium is: 


$$
\mathbf{H}_{i}=\mathbf{H}\left(U_{i} ; \boldsymbol{v}=\boldsymbol{\omega}=\mathbf{0}\right)=\left[\begin{array}{cccccc}
1 & 0 & 0 & 0 & z_{i} & -y_{i} \\
0 & 1 & 0 & -z_{i} & 0 & x_{i} \\
0 & 0 & 1 & y_{i} & -x_{i} & 0 \\
0 & -z_{i} & y_{i} & y_{i}^{2}+z_{i}^{2} & -x_{i} y_{i} & -x_{i} z_{i} \\
z_{i} & 0 & -x_{i} & -x_{i} y_{i} & x_{i}^{2}+z_{i}^{2} & -y_{i} z_{i} \\
-y_{i} & x_{i} & 0 & -x_{i} z_{i} & -y_{i} z_{i} & x_{i}^{2}+y_{i}^{2}
\end{array}\right]
$$

where $\mathbf{p}_{\mathbf{i}}=\left[x_{i} y_{i} z_{i}\right]^{T}$. The spatial stiffness matrix for fiducial registration is:

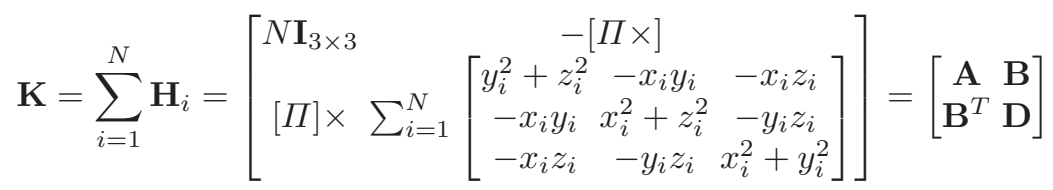

where $\Pi=\sum_{i=1}^{N} \mathbf{p}_{i}$ and the matrix $[\Pi \times]$ is the cross-product matrix, such that $[\Pi \times] \mathbf{u}=\Pi \times \mathbf{u}$. Equation $\square$ is a general expression of the stiffness matrix for our fiducial registration model where the fiducials are located in arbitrary positions.

When the centroid of the fiducials and the origin coincide, $\Pi=\mathbf{0}$ and $\mathbf{B}=\mathbf{B}^{T}=$ $[\mathbf{0}]$. In this case, the principal rotational stiffnesses are the eigenvalues of $\mathbf{K}_{V}=\mathbf{D}$. We recognize $\mathbf{D}$ as the inertia tensor for a system of $N$ point particles of unit mass (Meriam and Kraige [7]); thus, the eigenvalues are the principal moments of inertia and the eigenvectors are the principal axes. The fact that $\mathbf{B}=\mathbf{B}^{T}=[\mathbf{0}]$ is a very special case as it indicates that the rotational and translational aspects of stiffness are completely decoupled. In general, this decoupling is not possible (Lončarić [5]).

The principal translational stiffnesses are the eigenvalues of $\mathbf{A}$ which are $\sigma_{1}=\sigma_{2}=$ $\sigma_{3}=N$. The model predicts that fiducial registration is equally stiff with respect to translation in all directions, and that translational stiffnesses are independent of fiducial configuration.

\subsection{An Error Analysis of Fiducial Configurations}

We performed simulations similar to that described by Maurer et al. [6]. Four families of fiducial configurations parameterized by arc length $d$ were arranged on a sphere of radius $100 \mathrm{~mm}$ as shown in Figure 3 Target points were defined on all 3-D grid locations inside the sphere where the grid spacing was $5 \mathrm{~mm}$ in each direction. We used an isotropic effective fiducial localization error of magnitude $\mathrm{FLE}_{\text {eff }}=0.35 \mathrm{~mm}$, and a target localization error Maurer et al. ([6] $)$ of zero. The localized fiducials were simulated by adding the $\mathrm{FLE}_{\mathrm{eff}}$ to the target points. We then found the rigid transformation that registered the simulated fiducials to the targets using Horn's method [2] and computed the TRE for each target. This process of simulating localized fiducials, obtaining registrations, and computing the TRE was repeated 1000 times for each value of arc length $d=5,10,15, \ldots, 150 \mathrm{~mm}$.

We also computed the worst-case, or maximum, displacements predicted by stiffness analysis. Using Equation 7 we obtained the stiffness matrix of the fiducial configuration, and we computed the eigenvalues of Equation 3 to yield the principal rotational 

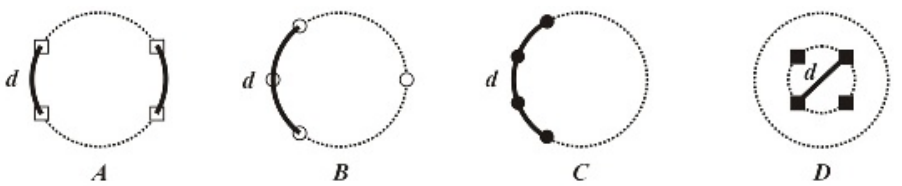

Fig. 3. Fiducial configurations on a sphere of radius $100 \mathrm{~mm}$ centered at the origin. In cases $A, B$, and $C$, the fiducials are located in the $x y$-plane. In case $D$, the fiducials are located in a plane parallel to the $x y$-plane. $d$ is the arc length on the great circle. In this view, +'ve $x$ is to the right and +'ve $y$ is to the top of the page.

stiffnesses. For each target we computed the equivalent stiffnesses using Equation 5 . With $\mu_{\mathrm{eq}, j}$ as the smallest equivalent stiffness, we used $\mu_{j}, \boldsymbol{\omega}_{j}$ and Equation 4 to compute the maximum displacement due to rotational compliance. In Equation 4 , the screw magnitude $\alpha$ was determined by considering the work done by the displacement. For a rotational displacement, the work done to displace a torsional spring of stiffness $\mu_{j}$ by a small amount $\alpha$ is $c=(1 / 2) \mu_{j} \alpha^{2}$ which for constant $c$ implies that $\alpha=\sqrt{2 c / \mu_{j}}$. Using Equations 2 and 7 it is easy to show that the term $\boldsymbol{\omega}_{j} \cdot \boldsymbol{v}_{j}=0$ and that the maximum displacement due to rotational compliance given by Equation 4 is $\rho_{j} \sqrt{2 c / \mu_{j}}$. By considering the work done, it can be shown that the maximum displacement due to translational compliance is $\sqrt{2 c / \sigma}=\sqrt{2 c / N}$. We took the total squared displacement to be $2 c \rho_{j}^{2} / \mu_{j}+2 c / N$. For the purposes of this article we found that choosing $c \approx 4 \mathrm{FLE}_{\mathrm{eff}}{ }^{2}$ produced excellent agreement with the numerical simulations; we are currently seeking theoretical justification for this value. The simulation and stiffness analysis results are shown in Figure 4.
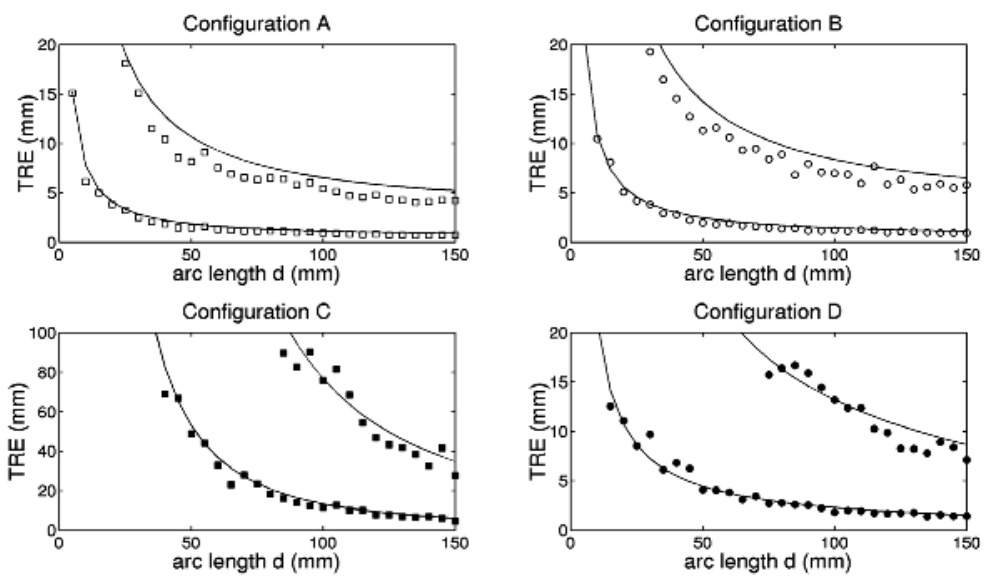

Fig. 4. Target registration error versus arc length between fiducials. On each graph, FLE $E_{\text {eff }}$ is 0.35 $\mathrm{mm}$ for the lower curve and $2 \mathrm{~mm}$ for the upper curve. Symbols indicate maximum TRE computed from simulations. Solid lines are maximum displacements computed from stiffness analysis. 


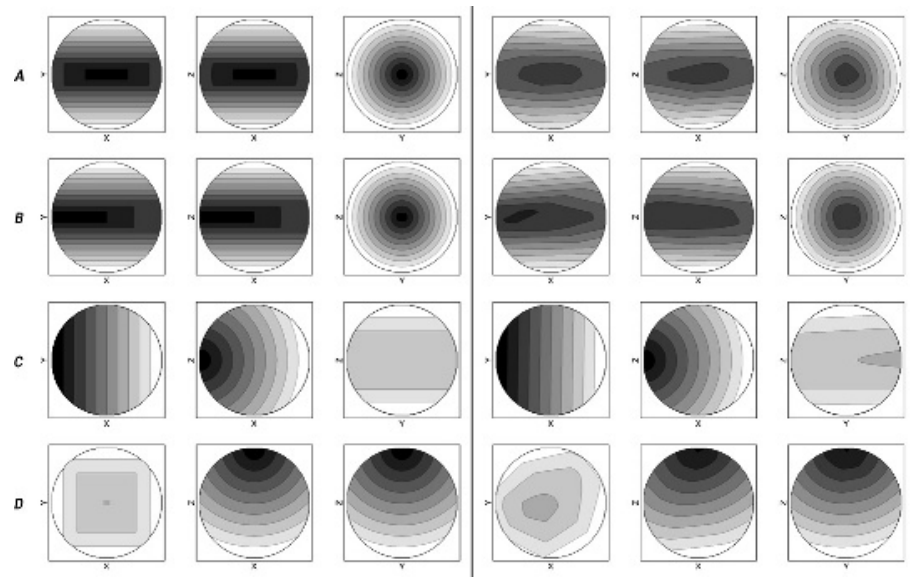

Fig. 5. Contour plots of normalized maximum displacements from stiffness analysis (left of solid line) and normalized maximum TRE from simulations (right of solid line). Results shown are for $d=50 \mathrm{~mm}$ and targets in the $z=0, y=0$, and $x=0$ planes. Registration errors increase from dark to light.

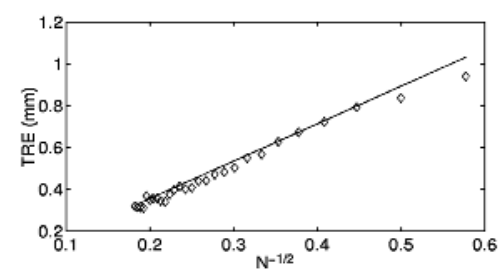

Fig. 6. Target registration error versus number of fiducials. Symbols indicate maximum TRE computed from simulations. The solid line indicates the maximum displacement computed from stiffness analysis.

\subsection{Error Isocontours}

In Maurer et al. [6] it was found that the mean TRE errors have elliptic isosurfaces. Stiffness analysis predicts that the maximum displacement resulting from rotational compliance is proportional to the distance from the most compliant screw axis; therefore, the isosurfaces should be cylindrical. Results from simulations and stiffness analysis for the four fiducial configurations are shown in Figure 5

\subsection{Error and Number of Fiducials}

Numerical simulations described by Maurer et al. [6] showed that the mean TRE was inversely proportional to the square root of the number of fiducials. Fritzpatrick et al. [1] predicted this relationship by deriving an approximate expression for the TRE. 
We can derive a similar relationship for the maximum displacement. Using Equations 3 and 7 and the fact that the trace of a matrix is equal to the sum of its eigenvalues (Horn and Johnson [3]), we find that

$$
\mu_{1}+\mu_{2}+\mu_{3}=\operatorname{trace}\left(\mathbf{K}_{V}\right)=\operatorname{trace}(\mathbf{D})=2\left(\sum_{i=1}^{N} x_{i}^{2}+\sum_{i=1}^{N} y_{i}^{2}+\sum_{i=1}^{N} z_{i}^{2}\right)=2 N R
$$

where $R$ is the average of the squared distance of the fiducials from the origin. Letting $\mu_{1}$ be the smallest rotational stiffness, it is easy to show that

$$
(\text { maximum displacement })^{2}=\frac{2 c \rho^{2}+2 c\left(2 R-\mu_{2}-\mu_{3}\right)}{2 N R-\mu_{2}-\mu_{3}}
$$

so we find that the maximum displacement is inversely proportional to $\sqrt{N}$.

We also computed maximum displacements using stiffness analysis and compared the results to the simulation described by Maurer et al. [6]. The simulation used from 3 to 30 fiducials evenly placed around the equator of a sphere of radius $100 \mathrm{~mm}$ with $\mathrm{FLE}_{\mathrm{eff}}=0.35 \mathrm{~mm}$. The results shown in Figure 6 show excellent agreement between the analytic predictions and the maximum TRE from the simulation.

\section{Discussion}

The use of stiffness analysis as a tool for evaluating registration stability was alluded to by Simon [12]. Instead of a frame-invariant analysis, he uses a principal-components analysis of a symmetric, positive semi-definite matrix to assess surface-based registration stability. Empirically, he found that registration accuracy improved when the noiseamplification index was minimized.

Our work is most closely related to the work described by Maurer et al. [6]. Many of the relationships for the maximum TRE - such as the independence of the rotational and translational error contributions, the independence of the translation error contribution on the fiducial configuration, the proportional dependence on $N^{-1 / 2}$, and the proportional dependence on the distance to the principal axes - are similar to those for the expected TRE. These results have been derived in a completely independent manner from Maurer et al. [6] and provide a sound theoretical basis for the analysis of registration accuracy.

One advantage of the spatial-stiffness framework is that we believe that the techniques can be extended to the case of rigid surface-based registration. This will require a more sophisticated spatial-stiffness model, perhaps incorporating surface-curvature effects. We anticipate that an analytic expression for the stiffness matrix will yield heuristics for optimizing registration point selection which will be much more efficient than genetic algorithms, such as those described by Simon [12]. The analytic expression may also help to justify other sampling heuristics, such as the uniform normal sampling heuristic described by Levoy [11]. Because the stiffness analysis is computationally inexpensive, there is the possibility that it can used online to interactively guide the user to better point selection during intraoperative registration of an image to a patient. 


\section{Conclusion}

We have described a method to predict the maximum target registration error for fiducial registration that closely matches the results from numerical simulations. This stiffness analysis approach is computationally efficient and we expect that it will generalize to surface-based registration.

Acknowledgments. This research was supported in part by the Institute for Robotics and Intelligent Systems, the Ontario Research and Development Challenge Fund, and the Natural Sciences and Engineering Research Council of Canada. We gratefully acknowledge Patricia McAllister (Department of Mechanical Engineering, Queen's University) for many helpful discussions regarding this research, as well as Amber Simpson and Richard Zanibbi (both from the School of Computing, Queen's University) for their careful proofreading of this article.

\section{References}

[1] J. M. Fitzpatrick, J. B. West, and C. R. Maurer, Jr. Predicting error in rigid-body point-based registration. IEEE Trans Med Imaging, 17(5): 694-702, Oct 1998.

[2] B. K. P. Horn. Closed-form solution of absolute orientation using unit quaternions. J Opt Soc Amer A, 4: 629-642, 1987.

[3] R. A. Horn and C. R. Johnson. Matrix Analysis. Cambridge University Press, 1985.

[4] Q. Lin, J. Burdick, and E. Rimon. A stiffness-based quality measure for compliant grasps and fixtures. IEEE Trans Robot Automat, 16(6): 675-688, Dec 2000.

[5] J. Lončarić. Normal forms of stiffness and compliance matrices. IEEE J Robot Automat, RA-3(6): 567-572, Dec 1987.

[6] C. R. Maurer, Jr., J. M. Fitzpatrick, M. Y. Wang, R. L. Galloway, Jr., R. J. Maciunas, and G. S. Allen. Registration of head volume images using implantable fiducial markers. IEEE Trans Med Imaging, 16(4): 447-462, Aug 1997.

[7] J. L. Meriam and L. G. Kraige. Engineering Mechanics: Dynamics. John Wiley and Sons, 1986.

[8] B. Mishra and N. Silver. Some discussion of static gripping and its stability. IEEE Trans Sys Man Cyber, 19(4): 783-796, Jul/Aug 1989.

[9] R. M. Murray, Z. Li, and S. S. Sastry. A Mathematical Introduction to Robotic Manipulation. CRC Press, 1994.

[10] T. Patterson and H. Lipkin. Structure of robot compliance. ASME J Mech Des, 115(3): 576-580, Sep 1993.

[11] S. Rusinkiewicz and M. Levoy. Efficient variants of the ICP algorithm. In Proc Int Conf 3D Digit Imaging Modeling, pages 145-152, 2001.

[12] D. A. Simon. Fast and Accurate Shape-Based Registration. PhD thesis, Carnegie Mellon University, Pittsburgh, Pennsylvania, Dec 1996. 